\title{
L'article scientifique : un produit culturel
}

\section{Odile Régent}

\section{(2) OpenEdition \\ Journals}

Édition électronique

URL : http://journals.openedition.org/asp/4017

DOI : $10.4000 /$ asp 4017

ISSN : 2108-6354

\section{Éditeur}

Groupe d'étude et de recherche en anglais de spécialité

\section{Édition imprimée}

Date de publication : 1 décembre 1994

Pagination : 55-59

ISSN : 1246-8185

\section{Référence électronique}

Odile Régent, «L'article scientifique : un produit culturel », ASp [En ligne], 5-6 | 1994, mis en ligne le 28 novembre 2013, consulté le 30 avril 2019. URL : http://journals.openedition.org/asp/4017 ; DOI :

$10.4000 / a s p .4017$

Ce document a été généré automatiquement le 30 avril 2019.

Tous droits réservés 


\title{
L'article scientifique : un produit culturel
}

\author{
Odile Régent
}

1 Le terme culture se trouve employé dans des sens très variés : on a pu opposer « high » et «low» culture, culture scientifique et culture littéraire, essayer de concilier toutes ces formes d'une réalité peut-être unique qui semblent s'opposer deux à deux. Pour tenter de clarifier un peu le débat, je propose le schéma suivant qui montre à quel point les différentes formes de culture sont imbriquées (voir figure 1).

Figure 1

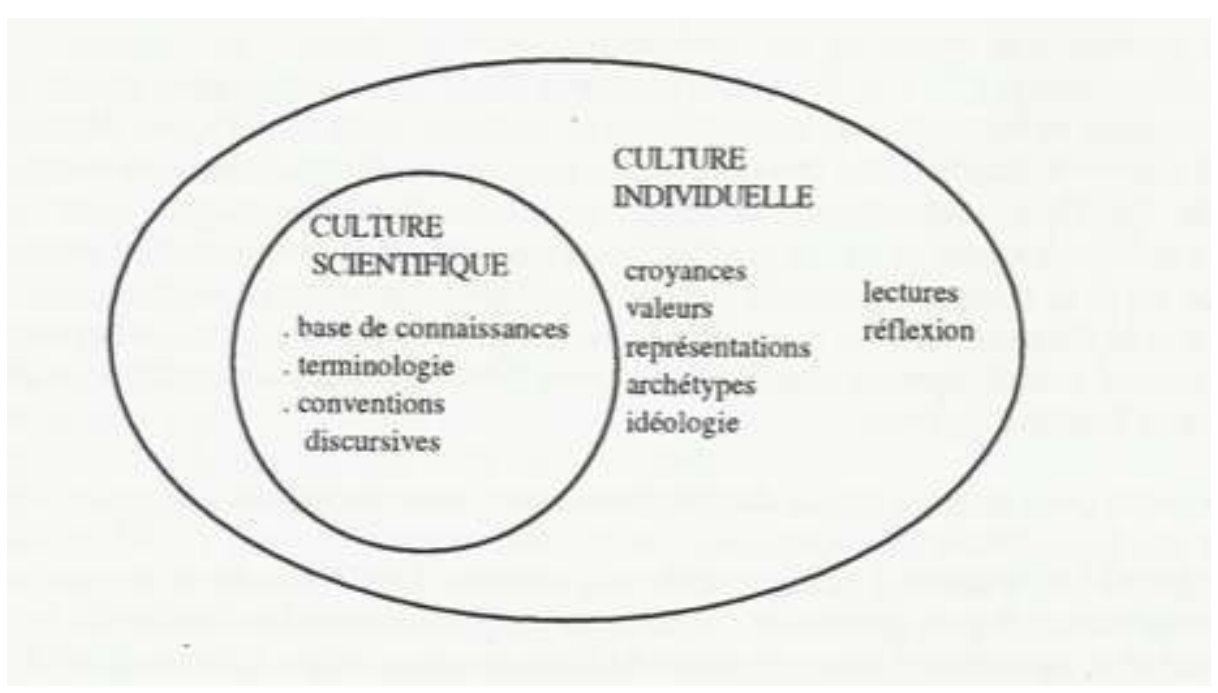

2 L'époque où les scientifiques se disaient détenteurs d'une culture uniquement fondée sur l'étude d'objets et le raisonnement logique est maintenant révolue. On admet que la personnalité du chercheur est déterminante dans le choix comme dans le traitement d'un objet scientifique: aucun objet n'est scientifique par essence, c'est le discours du chercheur qui lui confère cette caractéristique. L'hypothèse de Sapir (1967) d'une langue scientifique symbolique dont toutes les langues connues pourraient fournir des 
traductions (« une vérité scientifique est impersonnelle et n'est pas affectée par le moyen particulier qui l'exprime ») n'est plus défendable. La « langue scientifique » n'existe qu'au niveau de la terminologie: un objet qui devient scientifique par l'attention que lui portent les chercheurs reçoit une étiquette univoque et adaptée dans les langues connues. Au-delà de la terminologie, il n'y a que des discours scientifiques qui sont le fait de praticiens qui trient et transforment la réalité pour en faire des objets scientifiques en fonction de leurs opinions, de leurs idées reçues et du paradigme courant.

3 Les discours scientifiques sont donc étroitement dépendants du milieu et de l'époque dans lesquels ils se développent. L'ouvrage récent de L. Payer (1988) sur la médecine a popularisé cette idée en montrant comment les diagnostics et les traitements proposés pour les mêmes pathologies varient d'un pays à l'autre, d'une culture à l'autre. L'Américain garde l'esprit pionnier et attend du malade qu'il lutte, le français cartésien cherche une théorie et soigne le terrain avant la maladie. La représentation que le médecin a du malade et le malade du médecin sont ancrées dans les systèmes de valeurs et les croyances de chaque culture.

\section{La communication scientifique}

4 L'intertextualité joue un très grand rôle dans les sciences et particulièrement en médecine, discipline fondée sur l'observation où l'on a besoin de donner un poids statistique à des résultats en les confrontant à d'autres résultats. La communication est essentielle à l'intérieur de la communauté nationale et internationale. Pour la rendre possible, on a créé des modèles d'exposition du discours qui sont transculturels (Convention de Vancouver en sciences biomédicales). Les structures de surface des articles sont régies par des normes communes comme la structure IMRED (Introduction, Méthodes, Résultats Et Discussion) pour l'article original. Ces normes ne sont pas celles d'une langue scientifique mais des conventions arbitraires. La logique de l'exposition des résultats ne se superpose pas à la logique de la recherche. Il s'agit plutôt d'une manière de refaire l'histoire après coup. Néanmoins ces normes constituent un cadre éditorial commun et on peut donc parler d'une forme macrostructurelle d'exposition qui est transculturelle.

5 La culture scientifique n'est pas une entité unique, et on devrait plutôt parler de cultures scientifiques qui vont du niveau le plus élevé de spécialisation au niveau du public moyen qui s'intéresse un peu aux sciences. Les disciplines tendent à se subdiviser environ tous les 10 ans au fur et à mesure que la recherche avance et que le nombre de chercheurs augmente. On a ainsi plusieurs niveaux de communication : lorsqu'une nouvelle discipline apparait, elle ne concerne qu'un groupe très restreint de chercheurs souvent répartis sur plusieurs continents (les «invisible colleges»). Ils se communiquent leurs résultats dans des écrits qu'eux seuls comprennent et forment leur terminologie (communication ésotérique). Mais une discipline ne saurait survivre déconnectée des disciplines voisines, ni du reste de la communauté scientifique globale. Les chercheurs doivent donc être en mesure de communiquer leurs résultats dans des revues médicales générales ou scientifiques générales (communication exotérique). On peut ajouter aussi la nécessité actuelle de communiquer également avec le public au sens le plus large, car le financement de la recherche est en grande partie public et l'opinion attend que la communauté scientifique lui rende des comptes. Le chercheur étant également un citoyen doit se conformer à cette exigence. 
6 La nécessité pour le scientifique de communiquer à tous ces niveaux fait que la production écrite atteint des proportions considérables. On dénombre ainsi environ 25000 revues biomédicales dont 15000 « sérieuses » (Locke 1989), soit environ 2 millions d'articles par an, sans compter les revues scientifiques générales ni celles de vulgarisation. Cette inflation documentaire pose de nombreux problèmes de traitement de l'information et les éditeurs sont à la recherche de formes d'écrit plus compactes que l'article tel que nous le connaissons dans sa forme actuelle, persistante quoique déjà obsolète.

\section{La rhétorique contrastive}

7 Pour pouvoir communiquer efficacement dans un cadre transculturel, il est nécessaire d'être conscient des différences qui peuvent exister dans les articles produits dans des contextes de groupes restreints nationaux. À partir d'exemples tirés de revues biomédicales générales en français et en anglais, je tenterai de montrer comment les différences culturelles transparaissent dans un discours par ailleurs soumis à des normes rédactionnelles communes.

Remarque: la presse médicale française et l'anglo-saxonne ne sont pas vraiment comparables: l'anglais étant langue véhiculaire des sciences, beaucoup d'auteurs préfèrent publier en anglais dans des revues étrangères. La presse française est toutefois irremplaçable comme vecteur de communication de la communauté nationale et francophone. Elle contient surtout des écrits de caractère didactique: le spécialiste s'adresse au généraliste.

9 En publiant dans une revue générale, un auteur vise plusieurs objectifs : informer les publics ésotériques et exotériques (fonction cognitive ou référentielle) et négocier ou affirmer sa place dans le microcosme de sa spécialité et de la discipline en général (fonction affective, persuasion).

\section{L'énonciation}

10 Les procédés énonciatifs permettent une mise en scène de l'objet décrit : l'auteur se fonde sur les représentations qu'il a de cet objet et de l'image qu'il veut en donner. On a souvent noté l'effacement du je et l'abondance des formes impersonnelles et des nominalisations qui donnent la vedette à l'objet et laissent croire au lecteur que cet objet agit de luimême. À titre d'exemples :

Annales de Médecine Interne vol. $40 \mathrm{n}^{\circ} 3$

Études de cas

Mme Ginette $G$...41 ans est hospitalisée

Un homme de 20 ans sans antécédent pathologique est admis

L'interrogation retrouve fin avril la notion d'un érythème

A l'interrogatoire on apprend que

L'examen clinique note des adénopathies diffuses

MrK Jean Baptiste, 33 ans, ingénieur en informatique de nationalité ivoirienne était

hospitalisé le 201086

Fin août 1985, M.K ressentait une douleur

Le 309 85, une première intervention montrait la présence d'un hématome

New England Journal of Medicine vol. $322 \mathrm{n}^{\circ} 1$

Case records 


\begin{abstract}
A 29-year-old woman was admitted
There was a 10-year history of

Six months before admission abdominal pain developed

The patient was discharged on the 14th hospital day

At home the patient discontinued the course of

On physical examination the patient was

The urine gave a + test for protein

Specimens of blood, sputum and urine were obtained

On the following day the patient complained of "chills"
\end{abstract}

11 On note dans ces extraits comment l'objet d'observation, le patient, est réifié et comment les procédés énonciatifs utilisés font disparaître également le médecin qui procède à ces observations et écrit l'article. Le patient est sujet de verbes au passif en français comme en anglais. Les verbes à la voix active ressentait, discontinued, complained, sont en nombre limité et plus fréquents en anglais. Les nominalisations destinées à masquer les actants peuvent être d'un effet stylistique discutable : l'interrogation retrouve la notion de.. , the urine gave a + test. Si le patient américain semble moins passif, le français se voit souvent doté d'un prénom qui rappelle qu'on a affaire à un matériel humain. Le temps du récit est toujours le past en anglais alors que le français peut utiliser le présent ou l'imparfait de narration, procédé de type romanesque.

\title{
Le discours didactique
}

12 Le lieu de ce discours est l'éditorial et la mise au point (review). En français, ce discours se caractérise par des procédés qui relèvent du cours universitaire : abondance de questions rhétoriques, de définitions, et une mise en page qui souligne la structuration de cours par des procédés graphiques (intertitres, alinéas, caractères gras, italiques). L'objectif de ce discours est d'instruire et de donner des directives aux praticiens. Exemples :

Revue du Praticien vol. $39 \mathrm{n}^{\circ} 6$

Conduite pratique face à une maladie d'Alzheimer

début de l'article

- Tôt ou tard se posera la question du maintien à domicile

- ...le maintien est préférable chaque fois qu'il sera possible

- ...dans toute la mesure du possible il faut garder ces sujets chez eux

- Cette solution sera plus ou moins durable

fin de l'article

- ... un faux semblant pour masquer le refus de s'occuper d'autrui [...]. Car qui confère la dignité si ce n'est le regard de l'autre ? Robinson dans son île était-il un être digne avant l'arrivée de Vendredi ? Qui confère l'indignité si ce n'est le mépris ou le dégoût? Quelle est la dignité des mourants des rues de Calcutta avant que les sœurs de Mère Térésa les aient ramassés et conduits dans leurs établissements ? $\mathrm{Ne}$ la recouvrent-ils pas au même instant?

British Medical Bulletin vol $42 \mathrm{n}^{\circ} 1$

Management of dementia

début de l'article

- Can the Patient be Managed at Home?

- Most demented people are cared for by relatives at home (11) but the risk of breakdown is high (12)

- ... intensive, episodic interventions are all that can be offered (1-13-14)

- Will day Care Help?

fin de l'article

- Research on dementia [...] is at last widespread and advance in knowledge now carry some hope of effective treatment. 
- but the scale of need for personal care is unlikely ever to abate substantially.

- Already there is evidence that the demented are surviving longer

- ...So the challenge remains to put to better use the skill and resources we have

through more concerted and more sensitive responses.

13 Ces extraits sont tirés de numéros spéciaux de revues généralistes sur le même sujet : la maladie d'Alzheimer. Dès le début de l'article, l'auteur français se montre catégorique et prescriptif. L'auteur anglais appuie sa description de la situation sur des références bibliographiques : les chiffres entre parenthèses. Ces différences sont encore plus nettes dans les conclusions : l'auteur français en position dominante marque son autorité. Il se pose en détenteur du savoir et en gardien des valeurs morales du groupe. Ceci est très fréquent dans le corpus français où les auteurs ne ménagent pas leur peine pour faire la leçon à leurs collègues. On peut parler de stratégies de condescendance envers les pairs hiérarchiquement moins bien placés. Il est rare que des stratégies aussi explicites soient utilisées en anglais. Les marques que l'on peut observer sont plutôt du registre de l'innuendo.

On trouve en revanche des conseils et des prescriptifs dans les mémoires originaux ( research papers) plus souvent qu'en français où l'on termine parfois assez platement en reformulant une hypothèse interprétative. Les rédacteurs de revues anglo-saxonnes demandent expressément que la discussion débouche sur un message à la profession, " (it should) make sense of the findings ». Le BMJ signale comme l'un des motifs de refus des articles soumis « the absence of a message that is important to a general medical audience ».

\section{Implications didactiques}

Ces observations sont importantes car les Français qui souhaitent rédiger en anglais ne sont pas conscients de ces différences culturelles et comptent sur la transculturalité du discours scientifique pour rendre leurs articles simplement traduits acceptables dans une autre communauté. Or, des adaptations sont souvent nécessaires pour répondre aux attentes du lecteur d'une autre culture. Voici à titre d'illustration le plan d'un cours de rédaction scientifique pour des chercheurs de l'INSERM :

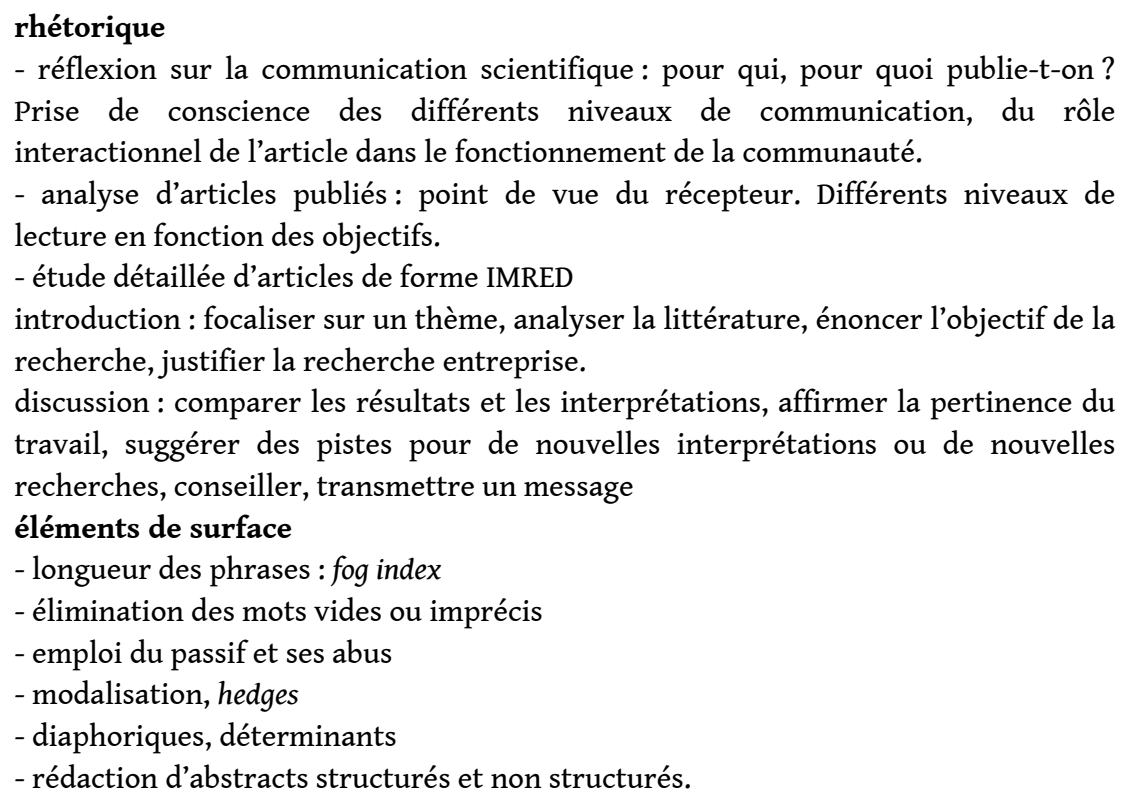


16

Les formes structurées tendent à se généraliser dans de nombreux types d'écrits : le texte est balisé d'intertitres obligatoires qui en structurent le contenu. Ces formes éliminent les modalisations et de nombreuses "politesses» (hedges) jugées jusqu'alors nécessaires (Myers 1989).

\section{BIBLIOGRAPHIE}

Lock S. 1989. « 'Journalology': Are the quotes needed ?». CBE Views 12/4.

Myers, G. 1989. « The pragmatics of politeness in scientific articles ». Applied Linguistics 10/1.

Payer, L. 1988. Medecine and Culture. New York : Holt.

Régent, O. 1992. «Pratiques de communication en médecine : contextes français et anglais ».

Langages 105.

Sapir, E. 1967. Le Langage. Paris : Payot.

\section{RÉSUMÉS}

L'auteur étudie l'article scientifique en tant qu'exemple de communication transculturelle au sein de la communauté internationale. Des exemples analysés, en provenance de revues de biomédecine publiées en anglais et en français, il ressort que les structures hiérarchiques des communautés nationales, ainsi que leurs croyances et valeurs, influent sur la manière dont est conduite la recherche, et dont en sont utilisés et transmis les résultats. Suit une discussion de possibles applications didactiques.

This paper investigates the scientific article as a case of transcultural communication within an international community. Analysis of examples drawn from French and English biomedical journals shows how the hierarchical structure of a national community, as well as its beliefs and values, influence the way research is carried out and its results used and transmitted. Didactic implications are discussed.

\section{INDEX}

Keywords : discourse analysis, scientific communication, culture

Mots-clés : analyse du discours, communication scientifique, culture

\section{AUTEUR}

\section{ODILE RÉGENT}

Odile Régent enseigne au CRAPEL, Université Nancy 2. Odile.Regent@univ-lorraine.fr 